\section{Perspectives from the American Society of Clinical Oncology 2014 Conference: breast cancer highlights}

Jennifer A Crozier ${ }^{1} \&$ Edith A Perez $z^{* 1,2}$

\author{
50th annual American Society for Clinical Oncology Meeting, Chicago, IL, USA, \\ 30 May-4 June 2014
}

The meeting included basic science and translational and clinical research in all specialties of hematology and oncology. From the multitude of abstracts presented, we will review the highlights regarding four abstracts discussing breast cancer.

The 50th annual American Society for Clinical Oncology meeting was held from 30 May to 4 June 2014 in Chicago, IL, USA. From the multitude of abstracts presented, we will review the highlights regarding four abstracts discussing breast cancer. One of the highlights and most controversial results was from the highly anticipated ALTTO trial [1]. ALTTO is a randomized Phase III trial comparing adjuvant anti-HER2 therapy concurrent with or after the completion of chemotherapy for early-stage HER $2^{+}$breast cancer. The trial enrolled 8381 women at 946 medical centers in 44 countries. The treatment arms compared 1 year of anti-HER 2 therapy with lapatinib alone $(\mathrm{L})$, trastuzumab alone $(\mathrm{T})$, their sequence $(\mathrm{T} \rightarrow \mathrm{L})$ or their combination $(\mathrm{L}+\mathrm{T})$. The primary end point was disease-free survival (DFS). The event rate was lower than the anticipated 850 event target, with 555 DFS events for the $\mathrm{L}+\mathrm{T}$ arm versus the $\mathrm{T}$ arm at 4.5 years of median follow-up, with patients doing better than anticipated. In fact, 4-year DFS rates of approximately $86 \%$ and overall survival (OS) rates of approximately $90 \%$ were observed. The trial did not meet its primary end points of showing that either dual blockade or sequential use of $\mathrm{T}$ followed by $\mathrm{L}$ significantly improved DFS. There was no significant difference in the 4-year DFS for L + T (88\%) versus T (86\%; hazard ratio: 0.84; 97.5\% CI: 0.7-1.02). Nor did it meets its primary end point, as DFS for the $\mathrm{T} \rightarrow \mathrm{L}$ arm was $87 \%$ versus $86 \%$ for the $\mathrm{T}$ arm (hazard ratio: $0.93 ; 97.5 \%$ CI: $0.76-1.13$ ). This is in stark contrast to the NeoALTTO trial in the neoadjuvant setting where $\mathrm{L}+\mathrm{T}$ resulted in a doubling in pathologic complete response (pCR) compared with $\mathrm{T}$ alone. With this difference comes an even larger concern regarding the significance of $\mathrm{pCR}$ and its relationship with long-term patient outcomes in HER2+ disease. Equally as important is the conclusion that the pCRs of neoadjuvant trials may not be so easily translated into the adjuvant setting.

The addition of bevacizumab (BV) to chemotherapy in the metastatic breast cancer setting has shown improvements in progression-free survival, but not OS, in several studies. Data evaluating BV in the adjuvant setting were reported by Miller et al. in the context of the Phase III E5103 study that enrolled 4994 patients with HER2- breast cancer [2]. Patients were assigned in a 1:2:2 fashion

'Department of Hematology \& Oncology, Mayo Clinic, Jacksonville, FL, USA

2Department of Cancer Biology, Mayo Clinic, Jacksonville, FL, USA

*Author for correspondence: perez.edith@mayo.edu

\section{KEYWORDS}

- adjuvant therapy

- ASCO • breast cancer

- chemotherapy • genomics

- highlights 
to one of three arms: standard therapy with doxorubicin and cyclophosphamide (AC) followed by weekly paclitaxel and placebo (arm A); AC followed by paclitaxel with BV throughout chemotherapy (arm B); and the same as arm B followed by ten additional cycles of BV after the completion of chemotherapy during radiation and endocrine therapy if appropriate ( $\operatorname{arm} \mathrm{C}$ ). Patients had a median age of 52 years with 64\% $\mathrm{ER}^{+}$and $27 \%$ lymph node-negative disease. In terms of receiving therapy, BV exposure was less than anticipated, with only $76 \%$ of patients in arm B and $45 \%$ of patients in arm C completing all planned BV therapy. Chemotherapyassociated adverse events included grade 4 neutropenia in approximately $20 \%$ and grade 3 or worse neuropathy in approximately $8 \%$ of patients across all arms. The primary end point was invasive DFS (IDFS), with the study meeting its predetermined power requirement with 430 IDFS events. At a median follow-up of 47.5 months, IDFS for arm A was 77\% (95\% CI: 70.9-81.2\%), arm B was 76\% (95\% CI: $71.5-79.8 \%$ ) and arm C was $80 \%$ (95\% CI: 77-82.5\%). The conclusion was that $\mathrm{BV}$ when added to $\mathrm{AC} \rightarrow$ paclitaxel in the adjuvant setting in HER2- patients provides no improvement in IDFS or OS and is more toxic, with increased adverse events.

Pagani et al. presented data regarding the hormonal therapy of premenopausal women with breast cancer [3]. The current standard of care of premenopausal women with $\mathrm{ER}^{+}$ breast cancer is to receive endocrine therapy with tamoxifen. Despite the benefit of aromatase inhibitors (AIs) in postmenopausal women, the question remains as to whether ovarian suppression and AIs would be superior to tamoxifen with or without ovarian suppression in premenopausal women. Both the SOFT and TEXT trials looked to address the question of tamoxifen versus AI for women who undergo ovarian suppression, and through an international collaboration, their data were analyzed together due to lower-thanexpected DFS event rates for each study. Taken together, the trials enrolled 4690 women with early-stage $\mathrm{ER}^{+}$breast cancer with or without axillary node involvement. Neoadjuvant and adjuvant chemotherapy was permitted. 57.4\% of patients received chemotherapy either after randomization in TEXT (34.3\% of all patients) or before randomization in SOFT $(23.2 \%)$. Patients were randomly assigned to exemestane $25 \mathrm{mg}$ daily (an AI) and ovarian suppression (EX-OS), tamoxifen $20 \mathrm{mg}$ daily and ovarian suppression (TX-OS) or tamoxifen alone (only in the SOFT trial). Ovarian suppression was achieved with the use of the gonadotropin-releasing hormone agonist triptorelin, oophorectomy or ovarian irradiation. The primary end point was DFS and secondary end points included breast cancer-free interval, distant recurrence-free interval and OS. DFS for EX-OS was $91.1 \%$ and for TX-OS was $87.3 \%$ (hazard ratio for recurrence, second invasive cancer or death: $0.72 ; 95 \% \mathrm{CI}$ : $0.60-0.85 ; \mathrm{p}<0.001)$. The current analysis reveals a $4 \%$ absolute improvement in breast cancer freedom with EX-OS compared with TX-OS. There was no difference in OS with the conclusion that the OS analysis is too soon at 68 months of follow-up. This study provides a reasonable alternative with exemestane and ovarian suppression, but best practice will not be established until comparison of tamoxifen alone is included (data are expected from the SOFT trial in December 2014).

The world of genomics is exploding throughout all cancer types. For HER2+ breast cancer, a novel predictive gene panel was presented by Perez et al. [4]. In order to better understand why certain patients develop tumor relapse after treatment with trastuzumab, an analysis of the North Central Cancer Treatment Group (NCCTG) N9831 adjuvant trastuzumab trial was conducted. Among the 1282 patients enrolled in the N9831 trial, whole-genome analysis technology was used in order to identify genes as well as key biological networks and pathways that are associated with relapse-free survival (RFS). For the trastuzumab-containing arms of the trial, six biological pathways (510 genes) associated with RFS were identified. Further analysis showed that 87 genes were prognostic for RFS for the patients in the trial arms receiving trastuzumab. This was further narrowed down to 14 genes that showed a significant difference in RFS when comparing the trastuzumab-containing arms with the chemotherapy-alone arm. This 14-gene panel was tested and validated repeatedly with the same predictive results. This integration of biology, genomics and clinical data provides a powerful predictive model for the benefit of trastuzumab. Additional validation of this work could have implications for patient care in the future. 
Financial \& competing interests disclosure

The authors have no relevant affiliations or financial involvement with any organization or entity with a financial interest in or financial conflict with the subject matter or materials discussed in the manuscript. This includes employment, consultancies, honoraria, stock ownership or options, expert testimony, grants or patents received or pending, or royalties.

No writing assistance was utilized in the production of this manuscript.

\section{References}

2 Miller K, O’Neill AM, Dang CT et al.

1 Piccart-Gebhart M, Holmes AP, Baselga J et al. First results from the Phase III ALTTO trial (BIG 2-06; NCCTG [Alliance] N063D) comparing one year of anti-HER2 therapy with lapatinib alone $(\mathrm{L})$, trastuzumab alone $(\mathrm{T})$, their sequence $(\mathrm{T} \rightarrow \mathrm{L})$, or their combination $(\mathrm{T}+\mathrm{L})$ in the adjuvant treatment of HER2-positive early breast cancer (EBC). J. Clin. Oncol. 32(5), Abstract LBA4 (2014).
Bevacizumab (BV) in the adjuvant treatment of HER2-negative breast cancer: final results from Eastern Cooperative Oncology Group E5103. J. Clin. Oncol. 32(5), Abstract 500 (2014).

3 Pagani O, Regan MM, Walley B et al. Randomized comparison of adjuvant aromatase inhibitor $(\mathrm{AI})$ exemestane (E) plus ovarian function suppression (OFS) vs tamoxifen $(\mathrm{T})$ plus OFS in premenopausal women with hormone receptor-positive $\left(\mathrm{HR}^{+}\right)$early breast cancer $(\mathrm{BC})$ : joint analysis of IBCSG TEXT and SOFT trials. J. Clin. Oncol. 32(5), Abstract LBA1 (2014).

4 Perez EA, Thompson EA, Anderson SK et al. Association of genomic analysis of immune function genes and clinical outcome in the NCCTG (Alliance) N9831 adjuvant trastuzumab trial. J. Clin. Oncol. 32(5), Abstract 509 (2014). 